\title{
TUTANKHAMEN'S TWO TOMBS: REPLICA CREATION AND THE PRESERVATION OF OUR CULTURAL HERITAGE IN THE DIGITAL AGE
}

\author{
L. Wong ${ }^{1 *}$, M. Santana Quintero ${ }^{2}$ \\ ${ }^{1}$ Getty Conservation Institute, 1200 Getty Center Drive, Suite 700, Los Angeles, CA 90049-1684, USA \\ LWong@getty.edu \\ ${ }^{2}$ Carleton Immersive Media Studio, Carleton University, 1125 Colonel by Drive, Ottawa, K1J 5B6 Canada - \\ Mario.Santana@carleton.ca
}

Commission VI, WG VI/4

KEY WORDS: Cultural Heritage, Conservation, Preservation, 3D Digital Documentation, Recording, Replicas

\begin{abstract}
:
There are two tombs of Tutankhamen both located in Luxor, Egypt: one in the Valley of the Kings, excavated into the Theban bedrock and decorated with wall paintings, dating from $1323 \mathrm{BCE}$; the other, installed $3 \mathrm{~km}$ away, opened in April 2014 and is considered to be an 'exact facsimile' of the original tomb. Tutankhamen's tomb is just one example of a cultural heritage site that has been replicated. This list is steadily growing as replicas are finding renewed favour fuelled by technological advancements in threedimensional recording, capture and fabrication which has enabled the production of highly accurate replicas in both physical and virtual form. Comparisons drawn between the two tombs of Tutankhamen - the original and the replica-aim to highlight the differing approaches of parallel preservation projects and intends to prompt questions surrounding the commissioning and use of replicas in the cultural heritage field and the growing role that 3D digital technology is playing in the preservation/conservation sector. Sites and cultural heritage organization are scrambling to be part of the 3D digital revolution. But, has the transition to 3D and the revival in replicas happened too quickly and at the expense of a site's other conservation needs? Is technology being used in the service of conservation and preservation or is it the other way around? How can those working with heritage balance the benefit of 3D technology with the overall conservation needs for a site? Using the example of Tutankhamen's two tombs this paper aims to provoke discussion on these topics.
\end{abstract}

\section{INTRODUCTION}

In 2009, the Getty Conservation Institute began a collaborative project with Egypt's Ministry of Antiquities to conserve the tomb of Tutankhamen in the Valley of the Kings, Luxor. This work was completed in early 2019. In parallel to this, a separate project by the Madrid-based Factum Foundation for Digital Technology in Conservation undertook high resolution image capture and laser scanning in the tomb in 2009. Five years later, in 2014, a replica of the tomb, considered to be an 'exact facsimile' by its creator, was installed at Carter House, $3 \mathrm{~km}$ away from the location of the original tomb. Today both tombs are open to visitors (see Figures 1-4).

The comparisons and contrasts drawn between the two tombs of Tutankhamen in this paper-the original and the replica-aim to highlight the differing approaches and narratives of overlapping conservation projects and intends to prompt questions surrounding the commissioning and use of replicas in the cultural heritage field and the growing role that $3 \mathrm{D}$ digital technology is playing in the preservation and conservation sector.

\subsection{The 3D 'Digital Revolution'}

The 1990s and early 2000s, heralded in a 'digital revolution' introducing a promising range of emerging tools for cultural heritage from the 3D to multimedia to the immersive world of virtual and augmented reality (Addison, 2001). This technology was received with great expectation as it offered the potential to advance the field in much needed areas of documentation, modelling and analyses, and the presentation and interpretation of heritage sites.

In the two decades that followed, technology in this area has continued to advance in terms of portability and reliability of instrumentation, accuracy and speed, and innovation in physical fabrication (i.e. computer numerical controlled milling and rapid prototyping) and immersive technology as well as the substantial lowering of costs. Sites, museums and cultural heritage organization are scrambling to be part of this 3D digital revolution and physical and virtual forms of replication are increasingly being utilized.

\subsection{The Role of Replicas in Cultural Heritage}

Tutankhamen's tomb is just one example of a cultural heritage site that has been 3D recorded and physically replicated. The rock art sites of Lascaux and Chauvet in France and Altamira in Spain are three other well-known cases where replicas now stand in for the original as the sites themselves have been closed for their protection. Many other examples exist and this list is steadily growing fuelled by the technological advancements in 3D recording, capture, physical fabrication, digital modelling and virtual and augmented reality.

No one can dispute the beneficial uses that replicas have enabled: allowing access to an otherwise closed or inaccessible site, increasing awareness and engagement, serving interpretive and educational purposes, and aiding researchers, to name only

\footnotetext{
* Corresponding author
} 


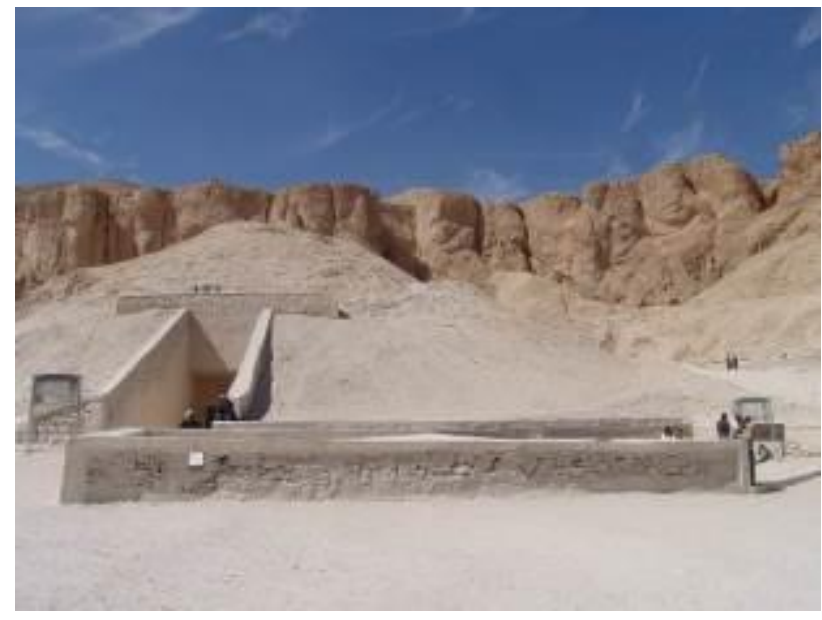

Figure 1. The entrance to the tomb of Tutankhamen, Valley of the Kings ( J. Paul Getty Trust 2009

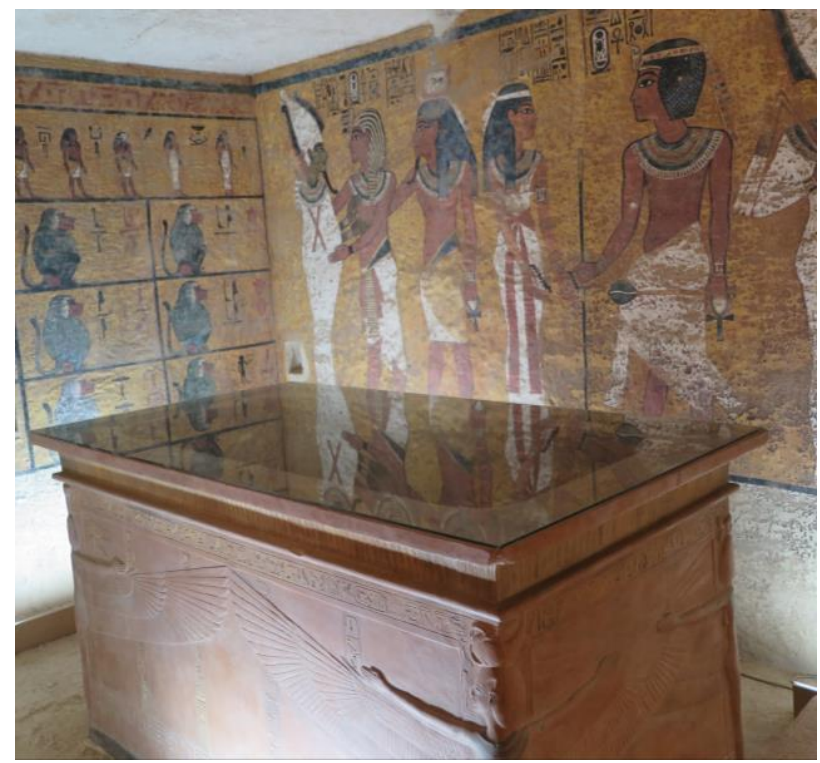

Figure 3. The tomb of Tutankhamen, Valley of the Kings (C) J. Paul Getty Trust 2015

a few. Today the potential role replicas can play continues to expand to now serve specific conservation objectives ranging from the creation of an archival and documentary record of a site for posterity to alleviating pressure from mass tourism by drawing tourists away from the actual site. The growing prominence and use of replicas in the cultural heritage field urges us to take a more careful and nuanced look at the commissioning, fabrication and application of these objects.

\subsection{Digital Documentation}

3D documentation, encompassing such recording and capture methods as laser scanning and photogrammetry, is one approach to collecting information and should be viewed as a component of the overall documentary record for a site. The specific role that $3 \mathrm{D}$ technology can play in the digital strategy for a site needs to be clearly defined and looked at in relation to the overarching conservation objectives.

Has the migration, adoption and reliance of 3D technology and

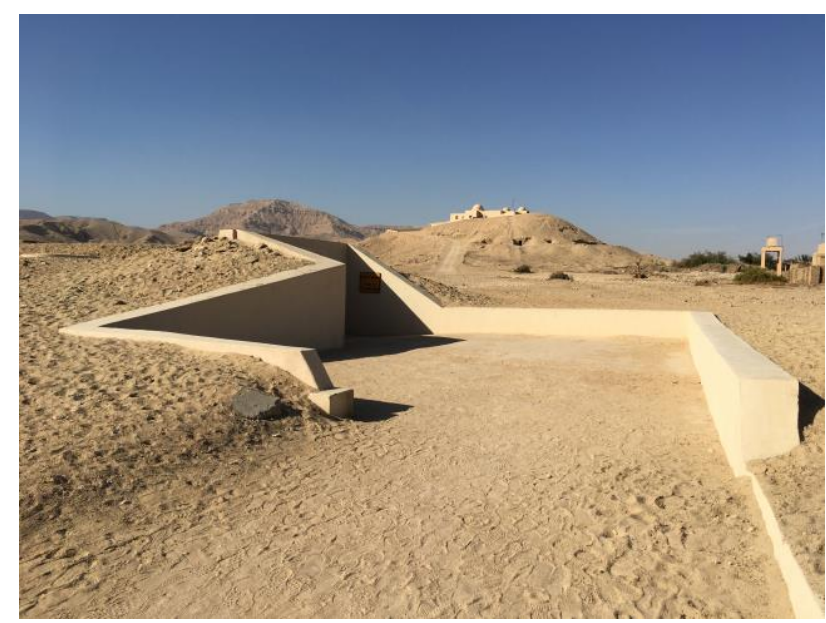

Figure 2. The entrance to the replica tomb of Tutankhamen at Carter House @ J. Paul Getty Trust 2009

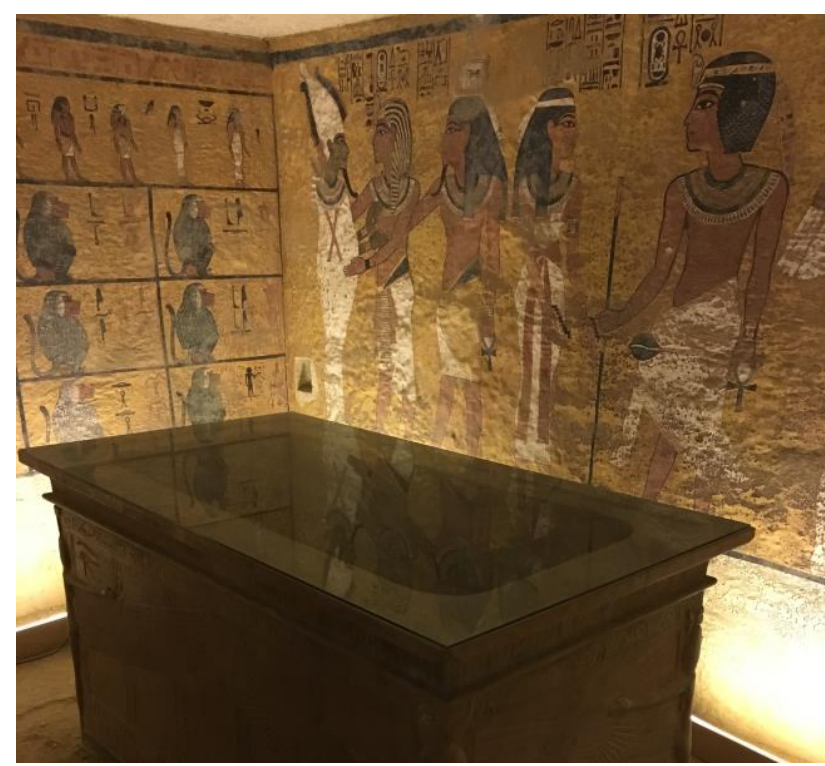

Figure 4. The replica tomb of Tutankhamen at Carter House (C) J. Paul Getty Trust 2019

the resurgence and renewed favour in replicas happened too quickly and at the expense of a site's other conservation needs? Is technology really being used in the service of conservation and preservation? Or, is it the other way around? Using the example of Tutankhamen's two tombs this paper aims to provoke discussion on these topics and to begin to look at how those working with heritage can balance the benefit of $3 \mathrm{D}$ technology and replica creation with the overall conservation needs of a site and where conservators and heritage recording experts can better align their goals and efforts in the interest of preserving cultural heritage.

\section{THE TWO TOMBS OF TUTANKHAMEN}

\subsection{Project Intent}

Tutankhamen's tomb was originally constructed (c. 1323 BCE) as a burial place and house of eternity for a deceased young pharaoh. 


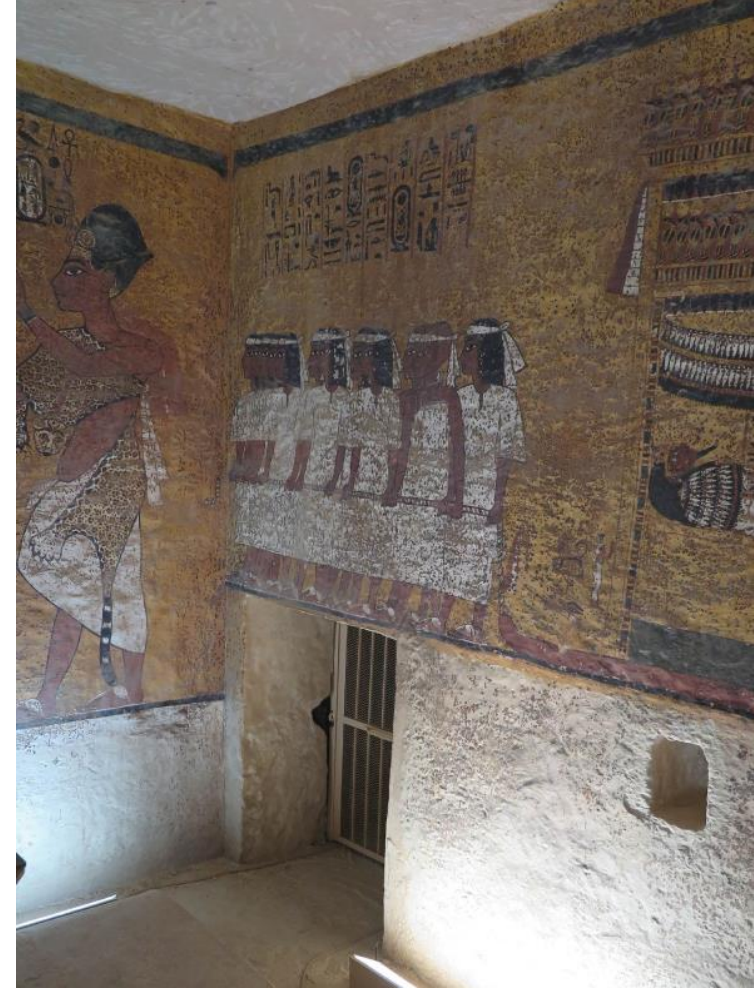

Figure 5. The northeast corner of the burial chamber of the tomb of Tutankhamen, Valley of the Kings (C) J. Paul Getty Trust 2015

During the collaborative project to conserve the tomb, between the Getty Conservation Institute and Egypt's Ministry of Antiquities, the principal focus was the design and implementation of an integrated conservation and management plan for the tomb and its wall paintings.

The main objectives of this project were to conserve the tomb and its wall paintings, by understanding original materials and techniques of execution and past physical history of the site in order to stabilize fragile paint layers and remove layers of dust from the tomb; determine ongoing threats to the tomb and implement actions to reduce future deterioration by improving environmental conditions; upgrading infrastructure (lighting, walkways, viewing platform, barriers, signage and ventilation); and, undertaking training of staff; and devising a program for maintenance, monitoring and visitation of the tomb.

The team was composed of Egyptologists to conduct background research on the tomb; environmental engineers to investigate microclimatic conditions; microbiologists to study the brown spots; heritage recording specialists to create a documentary record; architects and designers to upgrade the infrastructure; scientists to study the original materials of the wall paintings; and conservators to carry out condition recording and treatment and to train local conservators.

The project by the Factum Foundation for Digital Technology in Conservation, with a team composed of recording specialists, photographers, artists and conservators, aimed to digitally capture the original tomb by using a non-contact, reversible method to preserve the tomb for the future and to produce a high resolution replica. The resulting Tutankhamen replica was part of a larger Theban Necropolis Preservation Initiative

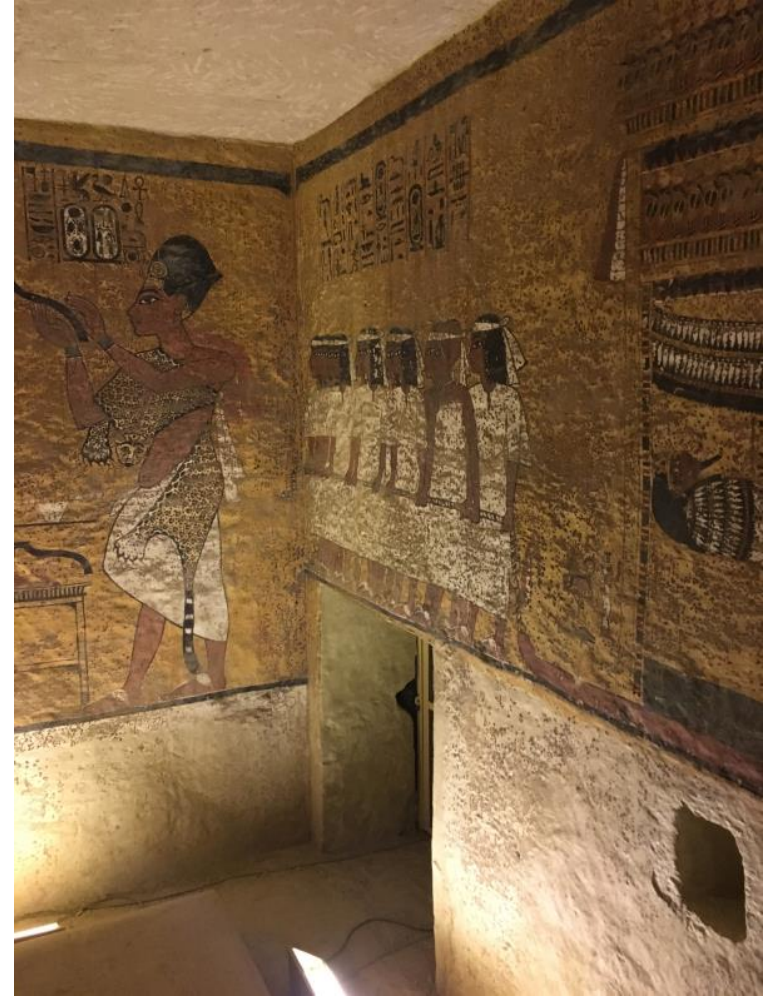

Figure 6. The northeast corner of the burial chamber of the facsimile of the tomb of Tutankhamen

(C) J. Paul Getty Trust 2019

(TNPI) between the Factum Foundation and the University of Basel, "to safeguard the tombs of the Theban Necropolis through the application of new recording technologies and the creation of exact facsimiles of tombs that are either closed to the public for conservation reasons or are in need of closure to preserve them for future generations". ${ }^{1}$

Both projects have the same end goal of preserving the tomb for future generations, however, the ways in which they approach conservation have conflicting narratives: one advocating for hands-on conservation, management of visitor numbers and improvements in environmental conditions of the original tomb, the other creating a documentary record and offering a facsimile tomb to visit, all of which is argued as a safer alternative to preserving the original tomb than traditional forms of conservation.

\subsection{Materiality}

The original tomb of Tutankhamen was made by ancient artisans over 3,000 years ago by chiselling into the limestone bedrock, coating the walls with clay mixed into a plaster, and painting images with a palette containing such pigments as yellow and red ochres and Egyptian blue and green.

The replica, in contrast, was made by modern day artisans using two-dimensional and three-dimensional high-resolution capture, then vacuum-adhering digitally printed images onto a milled and cast resin base that closely mirrors the surface contours of the original wall.

\footnotetext{
${ }^{1}$ http://www.factum-

arte.com/resources/files/fa/press_releases/web_tutankhamun_exhibi tion.pdf
} 


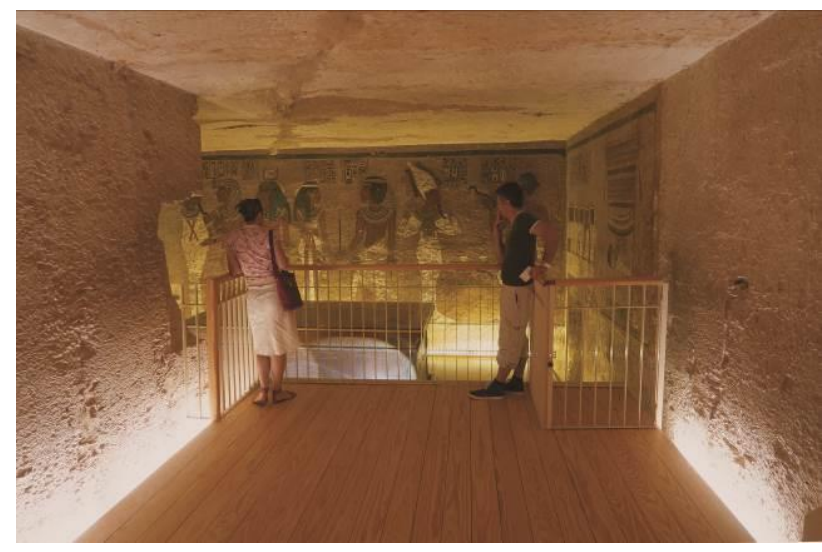

Figure 7. View of the antechamber looking north to the burial chamber (c) J. Paul Getty Trust 2015

Though the resulting replica has high fidelity in terms of its geometric form compared to the original with accurately replicated 3D surface texture, the digitally printed painted surface provides little information on material composition and traditional techniques of execution used (see Figure 5-6). Why was the decision made not to use materials similar to the original? Because of this difference in material composition, as the original and replica tomb age the differences between the two will become more and more apparent.

\subsection{Visitor Access}

The Egyptian authorities have not closed the real tomb so both tombs remain open. The viewing experience for the visitor in the replica tomb is remarkably similar to that of the actual tomb. Visitors are curiously constrained within the replica analogous to the original tomb: a wooden barrier prevents you from entering the burial chamber in both tombs (see Figures 7-8). Again, the goal seems to be for achieving accuracy but this time of the experience. Whereas the original tomb has crafted its visitors' experience according to an assessed need to prevent further mechanical damage to vulnerable wall paintings, the replica should be under no similar threat. Yet, the replica tomb of Tutankhamen has constructed barriers to visitors that imitate those of the original, toward a new goal of attempting to replicate the visitors' experience of the original tomb.

Supporters of the replica suggest that the tomb should be closed for its protection, while those involved in the study of the original tomb believe that the tomb can be kept open if the risks to the tomb are properly managed. How viable is it for the Egyptian authorities to close the original tomb? And, can visitors be persuaded to visit the replica instead?

\section{DISCUSSION}

\subsection{Authenticity}

The Tutankhamen replica is said by Factum Arte to redefine the relationship between originality and authenticity but at the same time it is not trying to deceive the visitor into believing it is the original. Article 13 in the Nara Document on Authenticity lists the various categories on which authenticity can be understood which include form and design, materials and substance, use and function, traditions and techniques, location and setting, and spirit and feeling which retain the cultural significance of

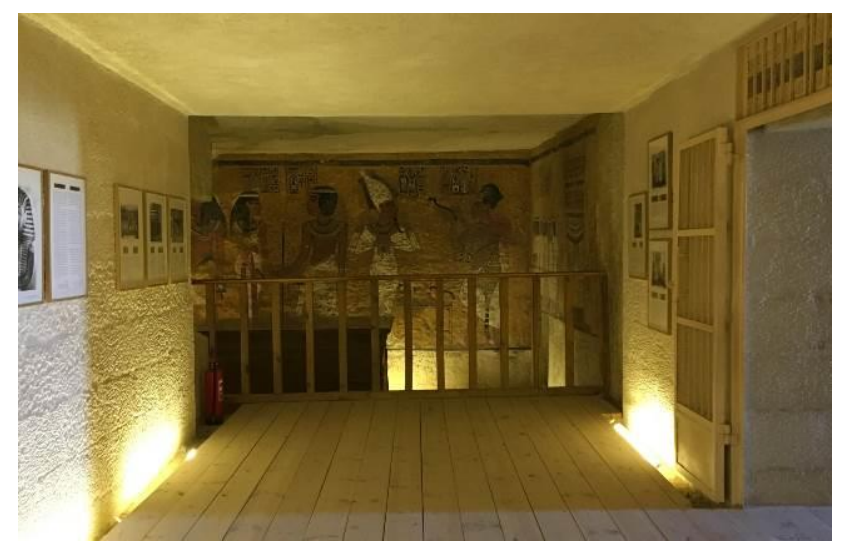

Figure 8. View of the antechamber looking north to the burial chamber in the replica tomb (C) J. Paul Getty Trust 2019

an object by providing reliable evidence of the past (UNESCO, 1994). Authenticity in the case of the Tutankhamen replica is primarily focused on replicating the form and design of the original tomb as well as its use and function by recreating the same visitor experience.

\subsection{Accuracy}

The replica places great emphasis on accuracy. The Factum Foundation claims that Tutankhamen was the highest resolution facsimile project to date that set new standards for replica fabrication. Using a 3D-scanning system custom built for this undertaking, they captured the tomb geometry to a 100 micron resolution. Additionally, recording of the painted surfaces with photographs was done 1:1 at 600-800 DPI (Foundation for Digital Technology in Conservation, 2009).

However, despite the quest for accuracy, the tomb was documented in 2009, prior to its conservation, when the paintings were covered with dust. Since then the paintings have been cleaned as part of the conservation work. Based on the timing of capture, the replica now preserves for posterity a record not only of the painting, but also of the obfuscation of the paintings by dust. Accuracy here is time dependent and despite the technological advancements in recording and capture methods the resulting documentation produces a static record which captures a single moment in time.

With the speed of technology advancements, how soon do our records become obsolete? Do we need to scan and capture again? Tutankhamen's tomb was documented again in 2017, following the completion of the conservation work, by Carleton Immersive Media Studio at Carleton University working in collaboration with the Getty Conservation Institute.

\subsection{Digital Strategies and Access}

The Factum Foundation has done an admirable job in handing over files to Egyptian authorities, creating a digital archive, providing equipment and transferring technological knowledge of their process through training. However, in general, many questions regarding digital strategies and access have emerged from the increase in $3 \mathrm{D}$ recording and capture of cultural heritage sites.

With significant post-processing work often required and the creation of diverse data formats and large file size there is a 
need to ensure metadata standards, technical rigour and transparency of how the data is produced and establish protocols for maintaining data in order to safeguard future access.

ICOM's International Committee for Documentation (CIDOC) says that "comprehensive approaches are still widely missing. The lack of digital strategies that encompass all digital activities of a museum results in inefficiencies, lack of defined priorities and unwisely spent resources."2

Few organizations have issued codes of ethics, principles and standards relating to the use of digital technology in cultural heritage and say little on the topic of replicas (Santana Quintero et al., Forthcoming 2019; Khunti, 2018).

The London Charter for the computer-based visualisation of cultural heritage seeks to establish principles in the research and communication of cultural heritage. ${ }^{3}$ Initiatives such as ReACH (Reproduction of Art and Cultural Heritage) launched in 2017 led by the V\&A in partnership with the Peri Charitable Foundation, and in which the Factum Foundation plays a role, "explores how to re-think our approach to reproducing, storing and sharing works of art and cultural heritage". ${ }^{4}$

Though the cultural heritage field is aware of the issues and need to create digital strategies without a clear path forward the push toward 3D technology and the increase in replica use can be viewed as brazen and irresponsible if the longevity of this amassed data is not assured.

\section{DECISION-MAKING}

The questions that have arisen from this exploration of the two tombs of Tutankhamen prompt us to also ask who should be part of the discussion and decision-making regarding replicas and the use of 3D technology. Presumably, the users: the owners, the educators, the researchers, the public and the many other stakeholders who will engage with the replica in some way and of course us, as professionals coming from the conservation and heritage recording fields.

However, Addison (2001) notes that "more often than not, those responsible for recording, preserving, and teaching about culture have not been part of this digital revolution". Today, the discourse on this topic has not been entirely democratic and equitable and our own professional self-interest overshadows other voices. Arguably, the creators of the replicas have had and will continue to have the stronger voice due to the technology gap that often exists and that we are the ones generally driving the discussion. The danger with this is that there is no one to oversee the use of 3D technology and replicas in the field of cultural heritage and it instead becomes controlled by those coming from the heritage recording sector. In this environment it becomes hard to maintain neutrality and in some cases the mantra of technology in the service of cultural heritage can be put in jeopardy.

Given the prevalence of the use of 3D technology and replicas

\footnotetext{
${ }^{2} \mathrm{http}: / /$ network.icom.museum/cidoc/working-groups/digitalstrategy-development/

${ }^{3} \mathrm{http}: / / \mathrm{www}$. londoncharter.org/objectives.html

${ }^{4}$ https://www.vam.ac.uk/research/projects/reach-reproductionof-art-and-cultural-heritage\#overview
}

in cultural heritage it is time for supranational organizations like UNESCO, ICOMOS and ICOM to take the lead in providing guidance in these areas with impartial and transparent involvement from the heritage recording field.

\section{CONCLUSION}

We are continually reminded about heritage at risk - the fear of destruction by war or terrorist acts; the threat of future loss from natural disasters, fire, development creep and neglect: all of these give us a sense of urgency and pushes us to act. But for whom? Why? And, how? In the case of Tutankhamen and other sites we need to ask these important questions.

Technology developments over the past two decades have allowed us to capture, record and replicate heritage sites faster, cheaper, and with increasingly higher resolution than ever before. But, there are now numerous digital initiatives all vying for attention and funding: the competition is fierce and the resources slim.

How could the conservation approaches between these two projects in Tutankhamen's tomb been better aligned? Why was a decision made to create a replica and who was involved in this process? The current trend to record cultural heritage using 3D technology though offering many benefits can also overshadow other conservation needs. Instead, the use of 3D technology and the decision of whether or not to produce a replica should be seen and discussed as part of the larger documentation record and conservation strategy for a site.

\section{ACKNOWLEDGEMENTS}

The authors wish to thank members of the Tutankhamen project team from Egypt's Ministry of Antiquities, the Getty Conservation Institute and Carleton Immersive Media Studio, Carleton University for their support.

\section{REFERENCES}

Addison, Alonzo C., 2001. Virtual heritage: technology in the service of culture in VAST '01 Proceedings of the 2001 conference on virtual reality, archeology, and cultural heritage held November 28 - 30, 2001, Glyfada, Greece, 343-354.

Factum Arte and The Foundation for Digital Technology in Conservation, The authorized facsimile of the burial chamber of Tutankhamun with sarcophagus, sarcophagus lid and the missing fragment from the south wall http://www.factumarte.com/resources/files/fa/press_releases/web_tutankhamun_exhibition .pdf.

Foundation for Digital Technology in Conservation, 2009. Factum Arte's Work in the Tombs of Tutankhamun, Nefertari and Seti I, March-May 2009 Report for the Supreme Council of Antiquities.

Khunti, R., 2018. The Problem with Printing Palmyra: Exploring the Ethics of Using 3D Printing Technology to Reconstruct Heritage. SDH, 2, 1, 1-12. DOI: 10.14434/sdh.v2i1.24590. 
Santana Quintero, M., Fai, S., Smith, L. Duer, A., Barazzetti, L., forthcoming 2019. Ethical framework for heritage recording specialists applying digital workflows for conservation, Int. Arch. Photogramm. Remote Sens. Spatial Inf. Sci.

UNESCO, 1994. Nara Document on Authenticity. Experts meeting, United Nations Educational, Scientific and Cultural Organisation, Paris. 